\title{
Broad-line radio galaxies: Old and feeble?
}

\author{
I. van Bemmel and P. Barthel
}

\author{
Kapteyn Astronomical Institute, PO Box 800, 9700 AV Groningen, The Netherlands
}

Received 21 August 2001 / Accepted 27 September 2001

\begin{abstract}
Far-infrared photometry of broad-line radio galaxies shows this class of AGN to consist of many hot and some cool infrared emitters, with peaks in their spectral energy distributions around $25 \mu \mathrm{m}$ or longward of $60 \mu \mathrm{m}$, respectively. Quantitative analysis indicates that this distribution relates to a substantial dispersion in the strength of the cool dust component: broad-line radio galaxies are relatively poor in large-scale dust. Possibly they have undergone a different merger evolution, or are relatively old AGN.
\end{abstract}

Key words. galaxies: active - infrared: galaxies - quasars: general

\section{Introduction}

Broad-line radio galaxies (BLRGs) were discovered and classified in the 1960's, as part of radio source identification programs (e.g., Matthews et al. 1964). Having optically visible host galaxies and optical spectra with broad recombination lines, they exhibit characteristics of both the narrow-line radio galaxies (NLRGs) and radioloud quasars (QSRs). Some of these BLRGs were identified with galaxies having a "brilliant starlike nucleus" (Matthews et al. 1964), the so-called N-galaxies (Morgan 1958). Their strong nuclear optical emission was subsequently discovered to experience reddening (Sandage 1973). It now appears that many BLRGs are hosted by such N-type galaxies (e.g., Hes et al. 1995). A thermal, accretion disk origin for their bright nuclear continuum has recently been established (e.g. Chiaberge et al. 2000). Unified models for radio-loud AGN postulate the presence of an opaque dust distribution (torus) in the radio axis equatorial plane, surrounding the continuum source and broad emission line region (Barthel 1989; Urry \& Padovani 1995). Within this framework BLRGs can be considered objects for which the line of sight is close to the torus edge. Thus the broad-line region is directly seen, but the central engine is still partially hidden from view.

Consistent with this picture, Cohen et al. (1999) found the optical spectra of several BLRGs to be a superposition of a reddened quasar spectrum and polarized (reflected) quasar emission. The reddening appears to increase for objects in which the polarization is higher, which can be interpreted as an increase in inclination. Using radio imaging and polarization data to address the aspect geometry, Dennett-Thorpe et al. (2000) found the class of BLRGs to consist of two groups. One contains

Send offprint requests to: I. van Bemmel, e-mail: bemmel@astro.rug.nl the objects which are powerful mis-aligned quasars in which the central engine is highly reddened. These objects are observed at relatively large inclination angles. The other consists of nearby, lower luminosity quasars, for which their proximity and low AGN luminosity combine to yield the observed broad-line radio galaxy. Like QSRs, these are observed at relatively small inclination angles. Prime example is 3C 111, for which superluminal behaviour in a one-sided radio jet - a typical QSR phenomenon - has been measured (Goetz et al. 1987).

A third way of addressing the aspect angle towards BLRGs is using infrared (IR) observations. The dust torus will absorb and re-emit the soft X-rays and UV emission of the AGN, and the emission will be anisotropic at wavelengths where the dust is optically thick. Radiative transfer models show that there is indeed a clear aspect angle dependence of the spectral energy distribution (SED) in the near- and mid-IR (Granato \& Danese 1994; Pier \& Krolik 1992). Consistent with the model predictions, QSRs are generally found to be stronger and somewhat warmer in the near- and mid-IR than NLRGs. This difference may well persist to wavelengths $\approx 60 \mu \mathrm{m}$ (van Bemmel et al. 2000). If BLRGs are mis-aligned and low-luminosity QSRs their IR SEDs are expected to be intermediate between those of QSRs and NLRGs.

The SED far-infrared (FIR) peak for QSRs and NLRGs is observed between 60 and $100 \mu \mathrm{m}$. However, IRAS data revealed several BLRG to display considerably warmer IR emission, indicating the presence of much hotter dust than in QSRs/NLRGs. Following the measurement of a pronounced $25 \mu \mathrm{m}$ peak in 3C 390.3 (Miley et al. 1984), more such cases were reported by Hes et al. (1995). In this paper we will study the FIR SED of the BLRG class, in particular the nature of the short wavelength peak. We will refer to the objects with a $25 \mu \mathrm{m}$ peak as hot, while the others will be called cool. 


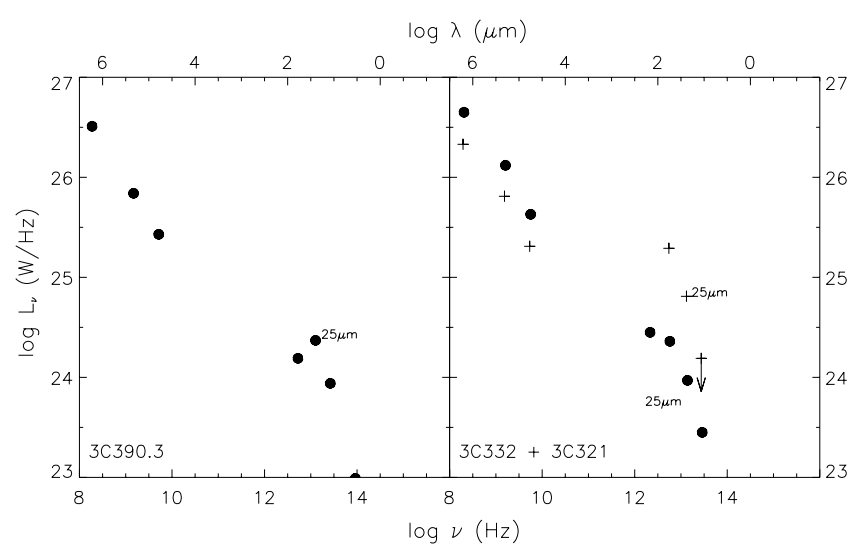

Fig. 1. Left panel: the restframe SED for the BLRG 3C 390.3 (hot BLRG). Right panel: the restframe SEDs for BLRG 3C 332 (filled circles) and NLRG 3C 321 (pluses), both cool objects. The $25 \mu \mathrm{m}$ points are marked in the plots. Errors are comparable to the size of the plot symbols.

\section{Observations and data reduction}

We carried out an ISO (Kessler et al. 1996) program, using ISOPHOT (Lemke et al. 1996), to obtain IR photometry for eight double-lobed BLRGs. The sample consisted of 3C 17, 3C 33.1, 3C 34, 3C 59, 3C $61.1,3 \mathrm{C} 330,3 \mathrm{C} 332$ and 3C 381, and was selected from Hes et al. (1995). Observations were made at $12,25,60,90$ and $160 \mu \mathrm{m}$, using the $\mathrm{P} 1, \mathrm{P} 2, \mathrm{C} 1$ and $\mathrm{C} 2$ detectors. Raster mode observing was applied, to avoid the known problems with chopping. The reduction of the $\mathrm{C} 1$ and $\mathrm{C} 2$ data followed the description in van Bemmel et al. (2000). The reduction of the $\mathrm{P} 1$ and $\mathrm{P} 2$ data is similar, the loss of redundancy is compensated by higher quality detectors, resulting in better data for the P-detectors. Unfortunately, 3C 34 was not detected at any wavelength, 3C 330 was not observed at $25 \mu \mathrm{m}$, and $3 \mathrm{C} 17$ was not observed at both 25 and $60 \mu \mathrm{m}$, which lead us to remove them from the sample. 3C 59 was rejected because of confusion with a nearby Seyfert galaxy. For two of the remaining objects the classification as a BLRG appears dubious. 3C 61.1 had been erroneously identified with a nearby radio-quiet QSO - hence its broad line spectrum; on the basis of a new high-resolution radio image it now appears to be an NLRG (Dennett-Thorpe et al. 2000). 3C 381 is reported to have broad Balmer line wings (Grandi \& Osterbrock 1978), but no literature spectra are available to confirm this. Dennett-Thorpe et al. (2000) conclude that 3C 381 is more likely to be an NLRG.

We extracted ISOPHOT archive data for BLRG 3C 109, and added five 3C BLRGs with IRAS detections at 25 and $60 \mu \mathrm{m}$, enlarging the BLRG database to a total of 8 bona fide objects, in the redshift range $0<z<0.3$. 3C 98 was included, having a $25 \mu \mathrm{m}$ detection in excess of its $60 \mu \mathrm{m}$ upper limit. 3C 234 and 3C 381 were added, in spite of their dubious BLRG nature (Cohen et al. 1999; Dennett-Thorpe et al. 2000). Lacking evidence that they are in fact NLRG, they will be treated as a BLRG, bringing the total to 10 objects.
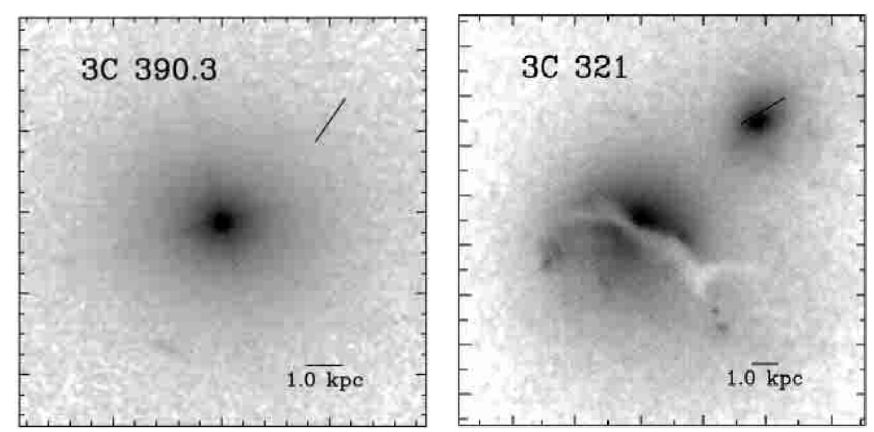

Fig. 2. Optical HST images of $3 \mathrm{C} 390.3$ (hot BLRG) and 3C 321 (cool NLRG). The black lines indicate the radio axis and the image scale respectively (de Koff et al. 1996).

It appears that 7 out of 10 BLRGs display a peak around $25 \mu \mathrm{m}$, with a positive spectral index ${ }^{1}$ between 60 and $25 \mu \mathrm{m}$. Three BLRGs show a small negative $\alpha_{25}^{60}$ value, which is still in marked contrast to NLRGs, displaying typical values $\alpha_{25}^{60} \approx-1.2$ (Hes et al. 1995). In order to compare objects of positive $\alpha_{25}^{60}$ with objects of negative $\alpha_{25}^{60}$, we supplement the BLRG sample with five 3C NLRGs of comparable redshift and radio luminosity. These NLRGs are obviously selected to have known 25 and $60 \mu \mathrm{m}$ flux densities from either IRAS (Golombek et al. 1988) or ISO (3C 61.1 - our measurements), and to have double-lobed radio morphologies.

Subsequently, the total sample of fifteen powerful FR II radio galaxies is divided into two sub-samples of hot and cool objects, depending on their value of $\alpha_{25}^{60}$, and regardless of their optical spectral classification. The hot subsample is defined as having a positive or flat spectral index, the cool subset has a negative spectral index. Typical SEDs for hot and cool BLRGs are shown in Fig. 1. To complement the IR SEDs and confirm the thermal nature of the IR emission, radio data have been extracted from NED and the literature. The two samples are listed in Table 1 . The luminosities have errors of $1 \%$ or less, and take redshift and K-correction effects into account.

\section{Results and analysis}

From Table 1 we see that both sub-samples are dominated by a different class: there are no hot NLRGs, and hardly any cool BLRGs. This is entirely in agreement with findings of Yates \& Longair (1989), Impey \& Gregorini (1993), Heckman et al. (1994) and Hes et al. (1995), and may provide an important clue to the nature of BLRGs.

Although incomplete, literature data did not reveal any differences between X-ray, optical, near-IR and radio properties of the sub-samples. With reference to Table 1 , we however establish a significant difference between the $60 \mu \mathrm{m}$ luminosities of the hot and the cool sample, while their $25 \mu \mathrm{m}$ and $178 \mathrm{MHz}$ luminosities are comparable. Thus, the hot broad-line objects must owe their classification as hot to their relative weakness at $60 \mu \mathrm{m}$. Assuming

\footnotetext{
${ }^{1}$ We assume $F_{\nu} \propto \nu^{\alpha}$.
} 
Table 1. Luminosity densities in $\mathrm{W} \mathrm{Hz}^{-1}$ at $25 \mu \mathrm{m}, 60 \mu \mathrm{m}$ and $178 \mathrm{MHz}$ for the hot and cool samples. Average luminosities for the hot and cool samples are given for comparison. The third column lists the nature of the object: B for BLRG and N for NLRG. 3C 234 and 3C 381 are marked B? in accordance with findings of Cohen et al. (1999) and Dennett-Thorpe et al. (2000). The fourth column lists the optical appearance: + for a disturbed host galaxy, - for a normal elliptical appearance, and ? if indecisive. We used $H_{0}=75 \mathrm{~km} \mathrm{~s}^{-1} \mathrm{Mpc}^{-1}$ and $k=\Lambda=0$ (i.e. $q_{0}=\frac{1}{2}$ ).

\begin{tabular}{|c|c|c|c|c|c|c|c|c|c|c|c|c|c|}
\hline \multicolumn{7}{|c|}{ Hot (peak around $25 \mu \mathrm{m}$ ) } & \multicolumn{7}{|c|}{ Cool (peak longward of $60 \mu \mathrm{m})$} \\
\hline Name & $z$ & ID & Type & $P_{25}$ & $P_{60}$ & $P_{178}$ & Name & $z$ & ID & Type & $P_{25}$ & $P_{60}$ & $P_{178}$ \\
\hline 3C 33.1 & 0.181 & $\mathrm{~B}$ & - & 24.41 & 24.36 & 26.93 & 3C 61.1 & 0.184 & $\mathrm{~N}$ & + & 23.97 & 24.59 & 27.39 \\
\hline 3C 98 & 0.030 & B & - & 23.19 & $<23.15$ & 25.92 & $3 \mathrm{C} 79$ & 0.256 & $\mathrm{~N}$ & + & 24.95 & 25.40 & 27.52 \\
\hline 3C 109 & 0.306 & $\mathrm{~B}$ & + & 25.78 & 25.36 & 27.60 & 3C 111 & 0.049 & $\mathrm{~B}$ & - & 24.01 & 24.16 & 26.20 \\
\hline $3 \mathrm{C} 234$ & 0.185 & $\mathrm{~B} ?$ & + & 25.29 & 25.14 & 27.38 & 3C 321 & 0.096 & $\mathrm{~N}$ & + & 24.84 & 25.30 & 26.31 \\
\hline $3 \mathrm{C} 382$ & 0.058 & $\mathrm{~B}$ & - & 23.78 & 23.82 & 26.11 & 3C 327 & 0.104 & $\mathrm{~N}$ & + & 24.80 & 25.16 & 26.96 \\
\hline $3 \mathrm{C} 390.3$ & 0.056 & B & - & 24.36 & 24.15 & 26.50 & 3C 332 & 0.152 & B & + & 23.99 & 24.36 & 26.63 \\
\hline $3 \mathrm{C} 445$ & 0.056 & B & - & 24.32 & 24.21 & 26.16 & 3C 381 & 0.161 & $\mathrm{~B} ?$ & $?$ & 24.31 & 24.48 & 26.92 \\
\hline & & & & & & & 3C 403 & 0.059 & $\mathrm{~N}$ & - & 24.17 & 24.47 & 26.27 \\
\hline Average & 0.124 & & & 24.45 & $<24.31$ & 26.65 & Average & 0.133 & & & 24.38 & 24.74 & 26.78 \\
\hline
\end{tabular}

the IR SED is dominated by thermal emission, this implies the absence of cool $(\sim 50 \mathrm{~K})$ dust in the hot objects. Hot $(\sim 150 \mathrm{~K})$ dust is present in all objects at a similar level, but it dominates the SED only in the hot sample. The visibility of broad emission lines must somehow be connected to relatively weak cool dust emission in this sample, and we proceed by addressing the origin of this cool dust.

There are two processes that heat dust up to $\sim 50 \mathrm{~K}$ in radio galaxies, namely the AGN (e.g., Hes et al. 1995) and star-formation. Detailed examination of the FIR SEDs of Seyfert galaxies (Rodríguez Espinosa et al. 1996), has indicated that the AGN strength is primarily reflected in the strength of the hot $(T \sim 150 \mathrm{~K})$ dust, i.e., the $25 \mu \mathrm{m}$ power. Prieto (2001) shows that the hot dust emission is correlated with the hard X-ray flux, whereas the cooler emission is not. This is consistent with our finding that $178 \mathrm{MHz}$ and $25 \mu \mathrm{m}$ luminosities, both reflecting the AGN power, are comparable between the hot and cool sample. We therefore exclude the AGN as the dominant heating mechanism. The FIR SED of the cool sample is comparable to that of dusty starburst galaxies, which generally have a peak in their SED around $60 \mu \mathrm{m}$ (e.g. Sanders \& Mirabel 1996). We therefore hypothesize that star-formation dominates the heating of cool dust to $50 \mathrm{~K}$, in which case the hot objects must have significantly less star-formation.

In the absence of $\mathrm{H} \alpha$ and/or UV data, we test this hypothesis using broad-band imaging data from the HST snapshot survey of the 3CR sample (de Koff et al. 1996). A three person blind test was done to classify the sample galaxies as non-interacting/dust-free, interacting/dustrich, or unclear: the results are summarized in Table 1. A clear relation is found between the visual appearance of the host galaxy and the shape of its IR SED. Hot BLRGs are predominantly normal, relaxed, dust-free elliptical galaxies, usually residing in sparse environments. The cool objects, however, display dust lanes, sometimes have close companions, and often show signs of interaction. The ubiquitous dust appears extended over tens of $\mathrm{kpc}$ and is mostly oriented perpendicular to the radio axis. In Fig. 2 two typical images for each sample are shown. Since the only disturbed cool BLRG 3C 332 has no clear dust lane, but is simply a relaxed elliptical with a nearby companion, we show cool NLRG 3C 321 for a more representative comparison. Both SEDs are included in Fig. 1. This striking difference in visual appearance indicates that many BLRGs are hosted by relaxed, dust- and gas-deficient galaxies, and provides, in addition, a natural explanation for the observed FIR SED difference.

\section{Discussion}

In line with trends reported by Hardcastle et al. (1998), some BLRGs such as superluminal, cool 3C 111 and optically variable, hot 3C 390.3 are undoubtedly low-power QSRs, observed at small inclination angles. Cohen et al. (1999) and Dennett-Thorpe et al. (2000) however point out that other BLRGs must be identified with critically inclined QSRs, i.e., radio sources observed close to or through their torus boundary. Since the dust content of a galaxy is unrelated to the orientation of its central radio source, a sizeable fraction of the BLRG population must constitute a class of its own. As a consequence, the clear line of sight towards the broad-line region in these hot BLRGs must be related to their dust- and gas-deficient host galaxies, either on large or on small scale. In the first case this implies that the obscuration of the broad-line region, generally assumed to occur in the circumnuclear torus (on sub-kpc scale), can also occur on much larger scales. Similar suggestions have been made for Seyfert galaxies (Malkan et al. 1998). However, there is no reason why the lack of cold dust on large scales does not extend into the nuclear regions, and thus the geometry of a central torus can be affected. Most likely, the torus is not solid, but exists of overlapping dense cool clouds. In the absence of a large supply of cold dust, these clouds may be smaller and less numerous, yielding low-extinction sight lines toward the central regions. 
As described above and has been reported elsewhere in much more detail (e.g., Heckman et al. 1986; Aretxaga et al. 2001), host galaxies of powerful radio sources frequently display signatures of ongoing or recent starformation, which is possibly connected to the onset of nuclear activity (O'Dea et al. 2001). Also several Seyfert galaxies and QSO hosts display such signatures, and age dating of their stellar population (e.g., González Delgado et al. 2001; Canalizo \& Stockton 2001) is strongly suggestive of an earlier, interaction-induced starburst phase, which produces and drives the fueling of the nuclear activity (Sanders et al. 1988; Barthel 2001). We observe no signs of this connection in the hot BLRGs. Nuclear activity and starbursts are generally assumed to be triggered by a major merger, and there are two ways to generate a relatively dust free radio galaxy. The first possibility is that such a radio galaxy forms from a single major dust-rich merger, the dust being removed during and immediately after the initial starburst phase. This scenario would be consistent with comparable AGN power and host galaxy luminosities for cool and hot objects. However, the dynamical timescales of large scale dust disks are of order $10^{9}$ years, about an order of magnitude larger than the maximum age of the radio source. A second possibility is that the hot BLRGs have been formed out of multiple dust-poor mergers. This results in comparable host galaxies and black hole masses, and naturally explains the lack of cold gas and dust in the hot objects (Haehnelt \& Kauffmann 2000).

Finally, if the onset of nuclear activity is preceded by a starburst phase, the ceasing of star-formation might be connected to the final evolutionary stages of the AGN. When all dust and gas has been dissipated, it is no longer possible to power star-formation and the active nucleus, and to sustain an obscuring torus. We note with interest that this scenario is consistent with the fact that the hot BLRGs in our sample are all relatively large double-lobed radio sources.

We plan to investigate these models using spectropolarimetric observations. The spectra of the underlying host galaxies will provide clues to the stellar population, and thus information on the merger history and age of the objects. Measurements of the cool gas content will also be relevant.

\section{Conclusions}

We have shown that hot objects, residing in dust-poor host galaxies, dominate the BLRG population. Their optical appearance shows little, if any, extended dust features, and their FIR SED is lacking emission from cool, star-formation related dust. This extended dust might contribute to the obscuration of the broad-line region in NLRGs. The lack of it in hot BLRGs can be explained either by a different merger history, or by their evolutionary stage.

Acknowledgements. The authors wish to thank Marshall Cohen and referee Chris O'Dea for detailed comments. Thanks are also due to Jane Dennett-Thorpe and Ronald Hes for their initial involvement in these BLRG investigations.

The NASA/IPAC Extragalactic Database (NED) is operated by the Jet Propulsion Laboratory, California Institute of Technology, under contract with the National Aeronautics and Space Administration. ISO is an ESA project with instruments funded by ESA member states (especially the PI countries: France, Germany, The Netherlands and the UK) and with participation of ISAS and NASA.

\section{References}

Aretxaga, I., Terlevich, E., Terlevich, R. J., Cotter, G., \& Díaz, A. I. 2001, MNRAS, 325, 636

Barthel, P. D. 1989, ApJ, 336, 606

Barthel, P. D. 2001, in FIRSED 2000 Proc., ed. I. van Bemmel et al., New Astron. Rev. [astro-ph/0104310]

Canalizo, G., \& Stockton, A. 2001, ApJ, 555, 719

Chiaberge, M., Capetti, A., \& Celotti, A. 2000, A\&A, 355, 873

Cohen, M. H., Ogle, P. M., Tran, H. D., et al. 1999, AJ, 118, 1963

De Koff, S., Baum, S. A., Sparks, W. B., et al. 1996, ApJS, 107, 621

Dennett-Thorpe, J., Barthel, P. D., \& van Bemmel, I. M. 2000, A\&A, 364, 501

Goetz, M. M. A., Preuss, E., Alef, W., \& Kellermann, K. I. 1987, A\&A, 176, 171

Golombek, D., Miley, G. K., \& Neugebauer, G. 1988, AJ, 95, 26 González Delgado, R. M., Heckman, T., \& Leitherer, C. 2001, ApJ, 546, 845

Grandi, S. A., \& Osterbrock, D. E. 1978, ApJ, 220, 783

Granato, G. L., \& Danese, L. 1994, MNRAS, 268, 235

Hardcastle, M. J., Alexander, P., Pooley, G. G., \& Riley, J. M. 1998, MNRAS, 296, 445

Haehnelt, M. G., \& Kauffmann, G. 2000, MNRAS, 318, L35

Heckman, T. M., Smith, E. P., Baum, S. A., et al. 1986, ApJ, 311,526

Heckman, T. M., O’Dea, C. P., Baum, S. A., \& Laurikainen, E. 1994, ApJ, 428, 65

Hes, R., Barthel, P. D., \& Hoekstra, H. 1995, A\&A, 303, 8

Impey, C., \& Gregorini, L. 1993, AJ, 105, 853

Kessler, M. F., Steinz, J. A., Anderegg, M. E., et al. 1996, A\&A, 315, L27

Lemke, D., Klaas, U., Abolins, J., et al. 1996, A\&A, 315, L64

Malkan, M. A., Gorjian, V., \& Tam, T. 1998, ApJS, 117, 25

Matthews, T. A., Morgan, W. W., \& Schmidt, M. 1964, ApJ, 140,35

Miley, G. K., Neugebauer, G., Clegg, P. E., et al. 1984, ApJ, 278, L79

Morgan, W. W. 1958, PASP, 70, 364

O'Dea, C. P., Koekemoer, A. M., Baum, S. A., et al. 2001, AJ, 121,1915

Pier, E. A., \& Krolik, J. H. 1992, ApJ, 401, 99

Prieto, A. 2001, in FIRSED 2000 Proc., ed. I. van Bemmel et al., New Astron. Rev. [astro-ph/0101103]

Rodríguez Espinosa, J. M., Pérez García, A. M., Lemke, D., et al. 1996, A\&A, 315, 129

Sandage, A. 1973, ApJ, 180, 687

Sanders, D. B., \& Mirabel, I. F. 1996, ARA\&A, 34, 749

Sanders, D. B., Soifer, B. T., Elias, J. H., et al. 1988, ApJ, 328, L35

Urry, C. M., \& Padovani, P. 1995, PASP, 107, 803

Van Bemmel, I. M., Barthel, P. D., \& de Graauw, M. W. M. 2000, A\&A, 359, 523

Yates, M. G., \& Longair, M. S. 1989, MNRAS, 241, 29 